\title{
Perméabilité intrinsèque et extrinsèque de couches minces ferromagnétiques et de multicouches ferromagnétique-isolant en fonction de la fréquence : comparaison expérience - modèle
}

\author{
A. BERTHAULT, F. DURBIN et J. RUSSAT
}

Commissariat a l'Energie Atomique, Centre d'Etudes de Bruyeres-le-Chatel, BP. 12, 91680 Bruyeres-le-Chatel, France

Soft ferromagnetic thin films are attractive materials for read/write head applications because they exhibit a high magnetic permeability in the hundred $\mathrm{MHz}$ range. By contrast, due to their low electrical resistivity, their processability at higher frequency is somewhat limited.

Using Maxwell equations and the geometry of the processed material, we have developed a theoretical model of the frequency-dependent magnetic permeability useful for multilayers design. We have distinguished different cases:

* extrinsic (measured) vs intrinsic permeability in magnetic thin films and magnetic-insulator multilayers,

* intrinsic vs extrinsic permeability in magnetic thin films, computed by the Newton iterative method.

Using the well-known Landau-Lifshitz model for high frequency permeability, we have compared experimental and theoretical results.

PERMEABILITE EXTRINSEQUE (MESUREE)

Considérons une lame mince d'épaisseur $e$ très inférieure aux dimensions latérales, d'un matériau de conductivité $\sigma$, magnétique de perméabilité complexe intrinsèque $\mu_{0} \mu_{\text {int }}\left(\mu_{0}=\right.$ $4 \pi \times 10^{-7} \mathrm{H} / \mathrm{m}$ ), placée dans un champ magnétique de pulsation $\omega$ dirigé selon l'axe $z$ $\left(\overrightarrow{\mathrm{H}}=H_{0} \mathrm{e}^{\mathrm{j} \omega t} \overrightarrow{\mathrm{z}}\right)$ et se propageant selon l'axe $y$. Calculons le champ $\overrightarrow{\mathrm{H}}$ à l'intérieur du métal, à partir des équations de Maxwell :

$$
\vec{\nabla} \times \overrightarrow{\mathrm{H}}=\overrightarrow{\mathrm{J}}=\sigma \overrightarrow{\mathrm{E}}, \vec{\nabla} \cdot \overrightarrow{\mathrm{B}}=0 \text { et } \vec{\nabla} \times \overrightarrow{\mathrm{E}}=-\mu_{0} \mu_{\text {int }} \frac{\partial \overrightarrow{\mathrm{H}}}{\partial t}=-\mathrm{j} \omega \mu_{0} \mu_{\mathrm{inz}} \overrightarrow{\mathrm{H}}
$$

On obtient,

$$
\vec{\nabla} \times \vec{\nabla} \times \overrightarrow{\mathrm{H}}=-\nabla^{2} \overrightarrow{\mathrm{H}}=\sigma \vec{\nabla} \times \overrightarrow{\mathrm{E}}=-\mathrm{j} \omega \sigma \mu_{0} \mu_{\mathrm{int}} \overrightarrow{\mathrm{H}}
$$

équivalent à,

$$
\Delta \overrightarrow{\mathrm{H}}-k^{2} \overrightarrow{\mathrm{H}}=0
$$

avec le nombre d'onde $k: \quad k=(1+\mathrm{j}) \sqrt{\frac{\pi v \mu_{0} \mu_{\text {int }}}{\rho}}=\frac{(1+\mathrm{j})}{\delta_{m}} \sqrt{\mu_{\text {int }}}$

où $\delta_{m}$ est le terme classique d'épaisseur de peau, $\rho=\sigma^{-1}$ la résistivité électrique et $v=\omega / 2 \pi$ la fréquence, variable balayée lors des acquisitions de spectres de perméabilité complexe.

Selon l'axe y normal à la lame, seule direction où l'amplitude de $H$ varie, nous avons donc à résoudre l'équation :

$$
d^{2} H_{y} / d y^{2}-k^{2} H_{y}=0
$$


En considérant l'origine des axes au centre de la lame, $H_{y}$ doit être une fonction paire et, en tenant compte des conditions aux linites $H_{y= \pm e / 2}=H_{0}$, la solution de l'Eq. (5) est :

$$
H_{y}=H_{0} \times \frac{\cosh (k y)}{\cosh \left\{\frac{k e}{2}\right\}}
$$

La perméabilité mesurée (ou extrinsèque) est déduite de l'induction moyenne dans la lame

d'épaisseur $e: \mu_{\text {ext }}=\frac{\langle B\rangle}{\mu_{0} H_{0}}=\mu_{\text {int }} \times \frac{\left\langle H_{y}\right\rangle}{H_{0}}=\frac{\mu_{\text {int }}}{e H_{0}} \times \int_{-e / 2}^{+e / 2} H_{y} d y=\mu_{\text {int }} \times \frac{\tanh \left\{\frac{k e}{2}\right\}}{\left\{\frac{k e}{2}\right\}}$

Afin de tenir compte de l'évolution en fréquence de la perméabilité intrinsèque, nous avons utilisé le formalisme de Landau et Lifshitz (L-L) [1] pour décrire complètement l'Eq. (7). L'accord entre les résultats expérimentaux et le modèle théorique défini par l'Eq. (7) est démontré en Fig. 1, pour un alliage ferromagnétique amorphe de composition CoFeSiB.

(a)

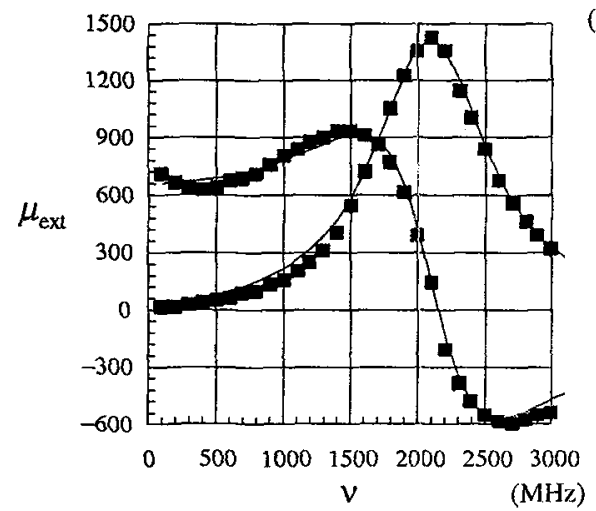

(b)

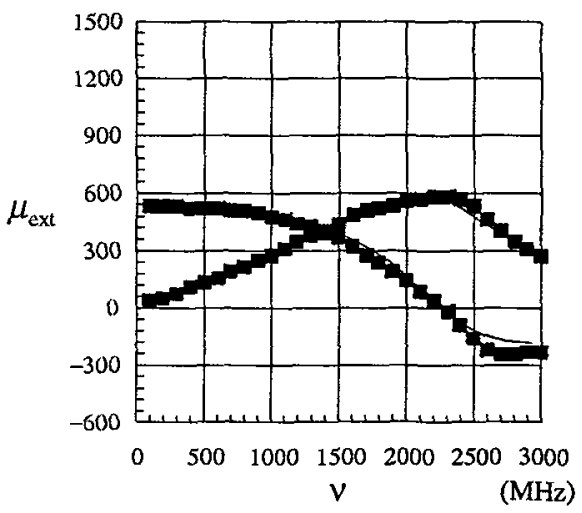

FiG. 1 : Spectres de perméabilité extrinsèque complexe expérimentaux $(\mathbf{G})$ et théoriques $(\longrightarrow$ ) pour un alliage ferromagnétique amorphe CoFeSiB épais de (a) 0,35 et (b) $1 \mu \mathrm{m}$.

\section{PERMEABILITE DE STRUCTURES MULTICOUCHES MAGNETIQUE-ISOLANT}

Nous ne traitons que le cas d'un nombre pair de couches magnétiques, le cas où il est impair se résolvant de manière similaire. Considérons un empilement de $2 N$ films magnétiques $\left(\mathrm{M}, \mu=\mu_{0} \mu_{\mathrm{int}}\right)$ d'épaisseur $e_{\mathrm{M}}$, et $2 N-1$ films isolants $\left(\mathrm{I}, \mu=\mu_{0}\right)$ d'épaisseur $e_{1}$, et d'épaisseur totale $e=2 N\left(e_{\mathrm{M}}+e_{\mathrm{I}}\right)-e_{1}$. Si nous choisissons la couche centrale isolante comme origine des axes $y$, par raison de symétrie, nous pouvons définir $2 N$ paires (Fig. 2) et l'induction sur les paires $[1, N]$ vaudra celle sur les paires $[-N,-1]$. 


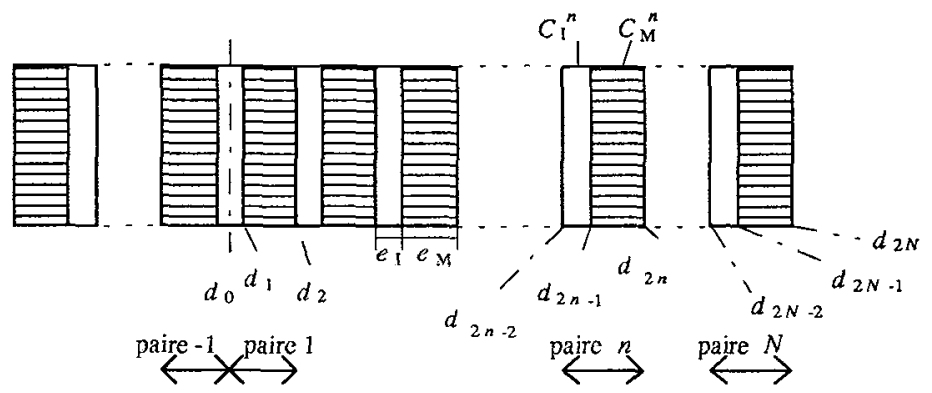

Fig. 2 : Schéma de la géométrie de la structure multicouche magnétique/isolant utilisée pour le calcul de $\mu_{\text {ex: }}$.

Les $\left\{d_{n}\right\}$ étant les positions des différentes interfaces ferromagnétique-isolant, nous avons : $d_{1}$ $-d_{0}=e_{\mathrm{I}} / 2, d_{2}-d_{1}=e_{\mathrm{M}}, \ldots, d_{2 n-1}-d_{2 n-2}=e_{1}$ et $d_{2 n}-d_{2 n-1}=e_{\mathrm{M}} \ldots$ Pour la $n^{\text {ème }}$ paire en partant du centre du composite, le champ dans la couche magnétique sera proportionnel à $H_{0}$, le champ à la surface. On montre que, par raison de symétrie, il doit être représenté par une fonction paire de la forme : $\quad H_{\mathrm{M}, y}^{n}=H_{0} \times C_{\mathrm{M}}^{n} \cosh (k y)$

et doit rester constant dans l'isolant : $\quad H_{I, y}^{n}=H_{0} \times C_{I}^{n}$

Les coefficients $C_{\mathrm{M}}^{n}$ et $C_{\mathrm{l}}^{n}$ sont déterminés par continuité du champ aux interfaces ; à la sur-

face, nous avons :

$$
H_{0} \times C_{\mathrm{M}}^{N} \cosh \left(\frac{k e}{2}\right)=H_{0}
$$

à l'interface ferromagnétique $(n) \leftrightarrow$ isolant $(n+1)$ :

$$
H_{0} \times C_{\mathrm{I}}^{n+1}=H_{0} \times C_{\mathrm{M}}^{n} \cosh \left\{k\left[n\left(e_{\mathrm{M}}+e_{\mathrm{I}}\right)-e_{\mathrm{I}} / 2\right]\right\}
$$

à l'interface isolant $(n) \leftrightarrow$ ferromagnétique $(n)$ :

$$
H_{0} \times C_{\mathrm{I}}^{n}=H_{0} \times C_{\mathrm{M}}^{n} \cosh \left\{k\left[(n-1)\left(e_{\mathrm{M}}+e_{\mathrm{I}}\right)+e_{\mathrm{I}} / 2\right]\right\}
$$

d'où : $\quad C_{\mathrm{M}}^{n}=\frac{1}{\cosh \left\{k\left[N\left(e_{\mathrm{M}}+e_{1}\right)+e_{1} / 2\right]\right\}} \times \prod_{p=0}^{N-n} \frac{\cosh \left\{k\left[(N-p)\left(e_{\mathrm{M}}+e_{1}\right)+e_{1} / 2\right]\right\}}{\cosh \left\{k\left[(N-p)\left(e_{\mathrm{M}}+e_{\mathrm{I}}\right)-e_{\mathrm{I}} / 2\right]\right\}}$

et

$$
C_{\mathrm{I}}^{n}=\prod_{p=0}^{N-n} \frac{\cosh \left\{k\left[(N-p-1)\left(e_{\mathrm{M}}+e_{\mathrm{I}}\right)+e_{\mathrm{I}} / 2\right]\right\}}{\cosh \left\{k\left[(N-p)\left(e_{\mathrm{M}}+e_{\mathrm{I}}\right)-e_{\mathrm{I}} / 2\right]\right\}}
$$

La perméabilité extrinsèque s'obtient par le calcul de l'induction magnétique moyenne dans

le composite d'épaisseur $e: \quad \quad \mu_{\mathrm{ext}}=-C_{\mathrm{I}}^{1} \frac{e_{1}}{e}$

$$
+\frac{2}{e} \sum_{n=1}^{N}\left\{C_{1}^{n} e_{1}+\mu_{\mathrm{int}} C_{\mathrm{M}}^{n} \frac{\sinh \left[k\left(n\left(e_{\mathrm{M}}+e_{1}\right)-e_{1} / 2\right)\right]-\sinh \left[k\left((n-1)\left(e_{\mathrm{M}}+e_{1}\right)+e_{1} / 2\right)\right]}{k}\right\}
$$

Remarquons que si les couches magnétiques sont en court-circuit par défaut d'isolation, le champ sera atténué par une couche magnétique d'épaisseur $2 \mathrm{Ne} e_{\mathrm{M}}$. Par dilution volumique on 
déduit $\mu_{\mathrm{ext}}$ :

$$
\mu_{\text {exL }}=\frac{(N-1 / 2) e_{1}+\frac{\mu_{\text {int }}}{k} \tanh \left(N k e_{\mathrm{M}}\right)}{(N-1 / 2) e_{\mathrm{I}}+N e_{\mathrm{M}}}
$$

En Fig. 3, nous comparons une simulation de spectres extrinsèques donnés par les Eqs. (15) et (16) à un spectre expérimental de 10 paires de $0,1 \mu \mathrm{m}$ de $\mathrm{CoZrNb}$ et $0,1 \mu \mathrm{m}$ de $\mathrm{SiO}_{2}$.

Notons que, curieusement, la perméabilité réelle prédite par l'Eq. (15) reste inférieure à celle obtenue en utilisant l'Eq. (16), dans la gamme des fréquences allant de 100 à $1000 \mathrm{MHz}$ (Fig. 3a). Ce fait inattendu est en contradiction avec l'opinion couramment acceptée que la structure en multicouches permet d'étendre la réponse en fréquence, par diminution de l'effet de peau.

On observe que l'allure du spectre expérimental (Fig. 3b) est mieux décrite par l'Eq. (16). Ce résultat pourrait être interprété par des effets de bord, dus aux dimensions latérales finies, ou par l'existence d'une conductance au travers de l'isolant, due à des inhomogénéités d'épaisseur.

(a)

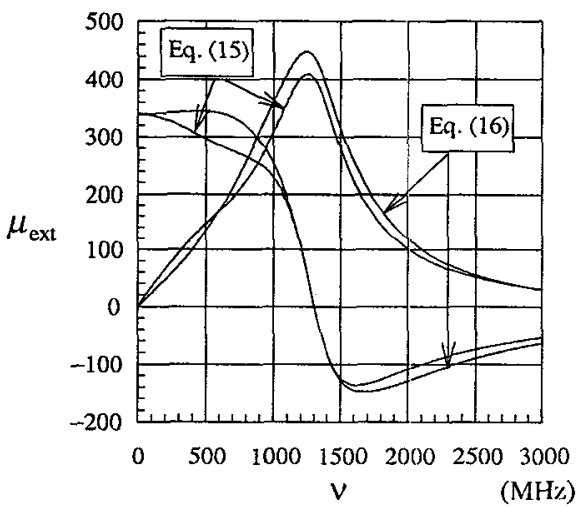

(b)

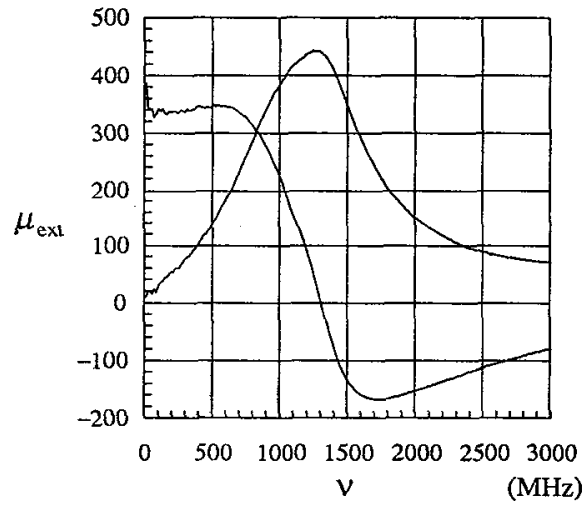

FIG. 3 : Spectres de perméabilité complexe extrinsèque d'une structure multicouche composée de 10 paires de $0,1 \mu \mathrm{m}$ de $\mathrm{CoNbZr}$ et $0,1 \mu \mathrm{m}$ de $\mathrm{SiO}_{2}$. La FIG. 3(a) représente deux spectres théoriques établis par l'Eq. (15) et l'Eq. (16) ; la FIG. 3(b) est le spectre expérimental correspondant.

\section{PERMEABILITE INTRINSEQUE:}

Récrivons l'Eq. (7) en effectuant les changements $\zeta=\frac{1+j}{2} \times \frac{e}{\delta_{m}}, z_{\text {mes }}=\zeta^{2} \mu_{\text {ext }}$ et $z=\zeta \sqrt{\mu}$ :

$$
z \tanh [z]-z_{\text {mes }}=0
$$

La résolution de l'Eq. (7) en $\mu$ équivaut donc à annuler, en $z=z_{r}$, la fonction $f$ définie par $f(z)=z \tanh [z]-z_{\text {mes }}$. Cette racine ne pouvant être calculée analytiquement, nous la déterminons numériquement par la méthode de Newton : en approximant $z_{r}$ par $z_{0}$, on a une meilleure approximation en $z_{1}=z_{0}-\frac{f\left(z_{0}\right)}{f^{\prime}\left(z_{0}\right)}$, puis en $z_{2}=z_{1}-\frac{f\left(z_{1}\right)}{f^{\prime}\left(z_{1}\right)}$ etc... Le $n^{\text {ième terme récur- }}$ rent est $z_{n}=z_{n-1}-\frac{f\left(z_{n-1}\right)}{f^{\prime}\left(z_{n-1}\right)}$. La suite $z_{0}, z_{1}, z_{2}, \ldots, z_{n}, \ldots$ tend vers la racine $z_{r}[2]$. 
(a)

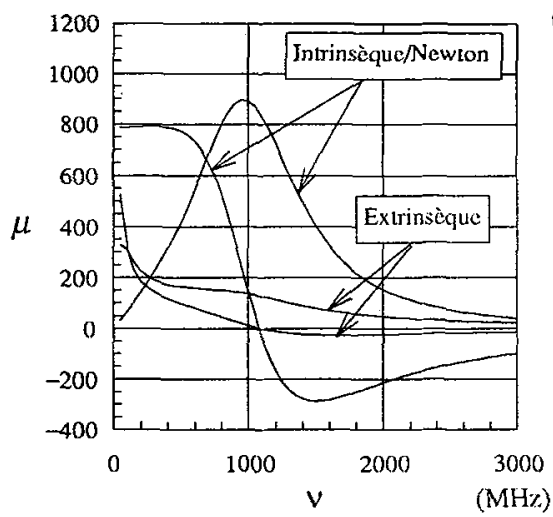

(b)

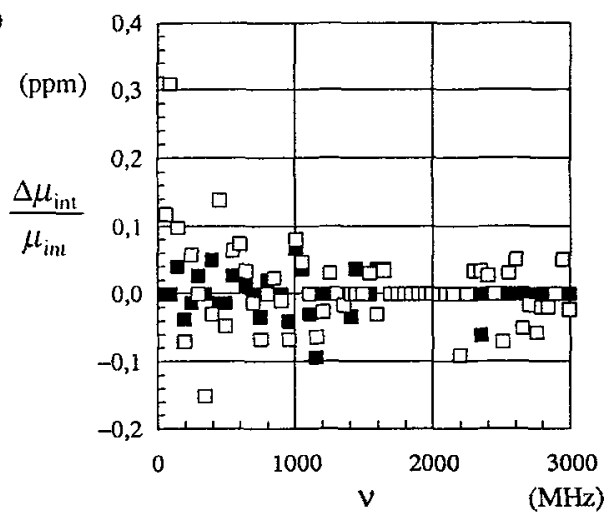

FIG. 4 : (a) Spectres de perméabilité complexe. Nous avons superposé le spectre intrinsèque, à celui obtenu par résolution de l'Eq. (7), ainsi que le spectre extrinsèque donné par application de l'Eq. (7) et (b) écart relatif entre le spectre intrinsèque théorique el celui calculé par résolution de l'Eq. (7) $\left(\square=\mu^{\prime} ; \square=\mu^{\prime \prime}\right)$.

Nous avons testé cette méthode de résolution avec des spectres théoriques de perméabilté. générés par l'utilisation des équations de L-L. Nous avons ensuite calculé le spectre extrinsèque, en utilisant l'Eq. (7) avec $\rho=1 \mu \Omega \mathrm{m}$ et $e=5 \mu \mathrm{m}$. Finalement, nous avons recalculé le spectre intrinsèque par la méthode de Newton, à partir des valeurs test $z_{0}=\zeta \sqrt{\mu_{\text {ext }}}$. Les résultats présentés en Fig. 4 nous permettent de comparer le spectre de perméabilité extrinsèque au spectre de perméabilité intrinsèque calculé par résolution de l'Eq. (7) en Fig. 4(a) et nous effectuons la différence entre la perméabilité intrinsèque théorique et celle calculée par la méthode de Newton en Fig. 4(b). Constatons que la résolution de l'Eq. (7) en exploitant les valeurs test bâties sur les valeurs $z_{0}=\zeta \sqrt{\mu_{\text {exl }}}$, permet d'accéder aux valeurs intrinsèques, avec une excellente précision $(\Delta \mu / \mu<1 \mathrm{ppm}$ !!), en au plus 5 itérations.

Le complexe $z_{r}$ est défini comme une fonction implicite de la variable $z_{\text {mes }}$ par la relation :

$$
f\left(z_{r}\right)=z_{r} \tanh \left[z_{r}\right]-z_{\text {mes }}=\phi\left(z_{r}\left(z_{\text {mes }}\right), z_{\text {mes }}\right)=0
$$

Cette fonction $\phi$ est égale à zéro ; sá dérivée par rapport à $z_{\text {mes }}$ au point $z_{r}$ vaut également zéro et s'écrit :

$$
\frac{d \phi}{d z_{\text {mes }}}=\left(\frac{\partial \phi}{\partial z} \times \frac{d z}{d z_{\text {mes }}}+\frac{\partial \phi}{\partial z_{\text {mes }}}\right)_{z=z_{r}}=0
$$

La grandeur $d z_{r} / d z_{\text {mes }}$ peut être considérée comme une sensibilité $\sigma$ de $z_{r}$ par rapport à $z_{\text {mes }}$ :

$$
\sigma=\frac{d z_{r}}{d z_{\text {mes }}}=-\frac{\partial \phi / \partial z_{\text {mes }}}{\partial \phi / \partial z}=\frac{1}{\tanh \left[z_{r}\right]+z_{r}\left(1-\tanh ^{2}\left[z_{r}\right]\right)}=\frac{1}{f^{\prime}\left(z_{r}\right)}
$$

Notons que $\left|f^{\prime}(z)\right|-1$, sauf pour $|z| \rightarrow 0$; il ne devrait donc pas y avoir d'amplification notable des erreurs de mesure dues à lá résolution.

Les complexes $z_{r}$ et $z_{\text {mes }}$ dépendant eux-même des paramètres $\mu_{\mathrm{exl}}, \mu, \rho$ et $e$, nous avons calculé formellement l'incertitude sur $\mu$, vis-à-vis des paramètres d'entrée $\mu_{\text {ext }}$, et $\rho$, que nous avons fait varier de $\pm 10 \%$ autour de leur valeur nominale, simulant ainsi une incertitude expérimentale. Les variations de $\rho$ ont peu d'influence sur le résultat final, l'essentiel étant dû aux variations de $\mu_{\mathrm{ext}}$ et $e$, qui seront les deux paramètres à connaître avec le plus de précision. Les résultats concernant l'incertitude sur $e$ sont donnés en Fig. 5. 


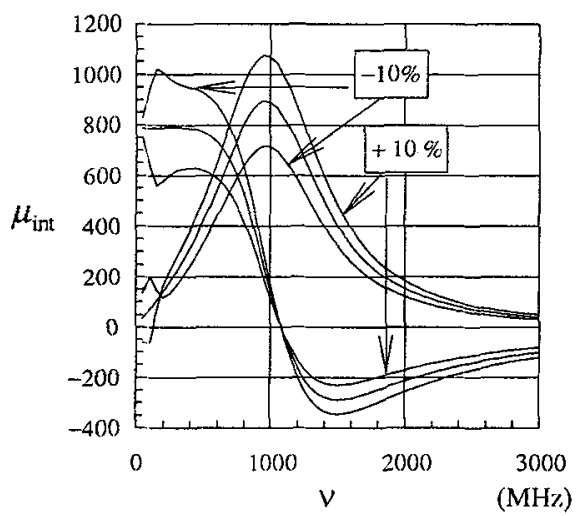

FIG. 5 : Spectres de perméabilité intrinsèjue complexe calculés avec une incertitude de $\pm 10 \%$ sur l'épaisseur.

\section{CONCLUSION}

Le modèle de perméabilité extrinsèque de couches minces est en accord avec l'expérience. L'interprétation des résultats obtenus pour les multicouches nécessite un approfondisement.

Le calcul de la perméabilité intrinsèque de couches minces par la méthode de Newton est performant sur une base théorique, si les paramètres géométriques et radio-électriques des échantillons sont connus avec précision.

Après validation, les modèles décrits précédemment devraient être utiles pour comprendre la physique des structures magnétiques en couches minces et servir de guide à leur élaboration.

\section{REFERENCES}

[1] A. H. Morrish, The Physical Principles of Magnetism, J. Wiley \& Sons, New-York, 1965. [2] A. Angot, Compléments de Mathématiques, MASSON ET Cic, Editeurs, 1972. 